\title{
Zur Geschichte der Akromegalie
}

Mit besonderer Berücksichtigung der Frühgeschichte im Kanton Glarus*

Von Hans Peter Schönwetter

«Nature in her ugliest mood conceived such a malady»
(In ihrer übelsten Laune schuf Mutter Natur diese Krankheit)
Harvey Cushing

Die Hypophyse ist diejenige endokrine Drüse, deren physiologische Funktion und deren Rolle beim Entstehen bestimmter Krankheiten zuletzt aufgeklärt werden konnte, ist doch das rein hypophysär bedingte Krankheitsbild der Akromegalie erst gegen Ende des letzten Jahrhunderts beschrieben worden.

\section{Was ist Akromegalie?}

Am 2. Mai 1927 berichtete das Magazin «Times» unter dem Titel «Hässlichkeiten», daß man in Paris, schon damals der Schönheitskonkurrenzen überdrüssig, nun auch Häßlichkeitswettbewerbe durchführe. Es wurde ironisch bemerkt, daß unter all den anwesenden Monstrositäten Mrs. Rosis Beavan, die «häßlichste Frau der Welt», fehlte. Sie hätte sicher den Sieg davongetragen. Schon am folgenden Tag verfaßte der weltbekannte Neurochirurg Harvey Cushing, damals Professor für Neurochirurgie an der Harvard University, einen Leserbrief, in dem er dem Herausgeber des Heftes vorwarf, auf Kosten der Unterhaltsamkeit unmenschlich zu sein. Die Geschichte dieser Frau hätte viel eher Tränen als Schmunzeln oder Gelächter verdient. Frau Beavan, eine früher gutaussehende Mutter von vier unmündigen Kindern, deren Gatte, ein Gärtner, wenige Jahre früher durch einen Unfall ums Leben gekommen war, wurde ein Opfer der als Akromegalie bezeichneten Krankheit. Das Leiden entstellte nicht nur ihren Körper und vor allem ihre Gesichtszüge, sondern verursachte ihr auch heftigste Kopfschmerzen und ließ sie fast erblinden. Um den Lebensunterhalt für die vier

* Meiner lieben Frau und zum 100. Geburtstag des Glarner Kantonsspitals. 
Kinder zu verdienen, mußte sie in der Kuriositätenschau des Zirkus der Ringling Brothers zwischen der dicksten Frau der Welt und dem «Armlosen Wunder» auftreten.

Wir wissen heute, daß die erst im Jahre 1886 von dem berühmten Pariser Neurologen Pierre Marie als Akromegalie bezeichnete Krankheit ihren Ursprung in einem Tumor der Hirnanhangsdrüse, d.h. in einem Hypophysenvorderlappenadenom, hat, das gesteigerte Mengen von Wachstumshormon produziert. Das im Überschuß produzierte Wachstumshormon ist neben der sekundär erscheinenden, tumorbedingten Zerstörung der Normalhypophyse, die den Ausfall der übrigen von ihr produzierten Hormone bedingt, verantwortlich für den überwiegenden Teil der Krankheitssymptome. Tritt die Erkrankung vor der Pubertät auf, d.h. vor Schluß der für das Längenwachstum verantwortlichen Wachtumszone (Epiphysenfugen) im Skelettbereich, so erfolgt ein abnormer Riesenwuchs oder Gigantismus.

Die heute noch vertretene Ansicht, die Diagnose der Akromegalie sei eine «Blickdiagnose», die durch weitere Diagnostik lediglich gesichert werden müsse, gilt nur für das Spätstadium. Das klassische Spätbild und seine Komplikationen sollten jedoch der Vergangenheit angehören. Durch Beachtung dezenter Frühsymptome - subjektiv geäußerte Beschwerden und objektive, klinisch oder laborchemisch nachweisbare Symptome - und durch sofortige radioimmunologische Wachstumshormonbestimmung sollten frühzeitige Erkennung und rechtzeitige Behandlung möglich sein.

Im Frühstadium kommt es meist vor Auftreten der charakteristischen, phänotypischen Veränderungen im Gesicht und im Bereich der Akren (Abb. 3 und 6) zu einer Überfunktion der Hautanhangsgebilde mit einer Neigung zu exzessivem unmotiviertem Schwitzen, das meist in allen Stadien ein typisches Zeichen für Aktivität und Progredienz des Prozesses ist. Es kommt zu Menstruationsstörungen, Libidoverminderung, Potenzstörungen und bei etwa 14\% der Kranken zu einer Galaktorrhoe. Durch Zunahme des Bindegewebes mit Weichteilveränderungen kommt es zu Schwellungen der Hände (Ringe müssen erweitert werden!) und der Füße, insbesondere des Fersenpolsters (größere Schuhnummern werden notwendig!), zur Verdikkung der Lippen und Nasenweichteile, zur Makroglossie und als Folge zu kloßiger, nasaler Sprache sowie zur Betonung der Hautfalten. Mit zunehmender Erkrankungsdauer kommt es zur Betonung der Supraorbitalwülste und zur Progenie, zur Brustkyphose mit nach vorne geneigtem Kopf, der sogenannten «Fighterstellung», und zu schwerfälligem Gang. Der Gesichtsausdruck ändert sich; er wird grobschlächtig mit einem drohenden, oft 
unheimlichen Anstrich. Schwere Knochen-, Gelenk- und Wirbelsäulenveränderungen durch periostales und appositionelles Knochenwachstum führen zu neuralgischen Beschwerden bis zu druckbedingten Lähmungen, teilweise mitbedingt durch eine hypertrophe Neuropathie, durch perineurale Fibrose und vermehrte extrazelluläre $W$ assereinlagerung. Jeder zweite akromegale Patient leidet unter einem Karpaltunnel-Syndrom.

Im Rahmen der Splanchnomegalie, d.h. Vergrößerung aller innern Organe, kommt lediglich der Kardiomegalie pathogenetische Bedeutung zu. Durch Vermehrung der Muskelmasse über die Zunahme von Zellgröße und -zahl werden Herzgewichte von über $1000 \mathrm{~g}$ erreicht. Dies führt neben einer progressiven Kardiovalvulopathie zu einer relativen Koronarinsuffienz mit allen Zeichen und Komplikationen der koronaren Herzkrankheit bis zum Herzinfarkt. Ca. $74 \%$ der akromegalen Patienten weisen eine euthyreote Struma auf, die wachstumshormonbedingt ist und deshalb nicht auf Behandlung mit Schilddrüsenhormonen anspricht und die bei $10 \%$ der Patienten vor der Diagnose der Akromegalie zur Strumektomie führte.

Die röntgenologischen Veränderungen, wie Hyperostosis frontalis, Vergrößerung der Sinus frontales, Pneumatisation der Mastoidzellen, Stumpfwerden des Kieferwinkels und Progenie, unvollständiger Zahnschluß und Auseinanderweichen der Zähne, Veränderungen der Wirbelsäule und hypertrophe Arthrose der großen Gelenke, sind unspezifisch, dokumentieren jedoch die schweren körperlichen Veränderungen. Bei $80 \%$ der akromegalen Patienten werden jedoch zum Zeitpunkt der Diagnosestellung dezente Sellaveränderungen (z.T. nur vom Neuroradiologen zu erkennen) nachgewiesen. Bei den restlichen $20 \%$ der Patienten ohne Sellaveränderungen kann man heute mit speziellen neuroradiologischen Techniken (u. a. KranioComputer-Tomometrie mit Kontrastmittelgabe) auch rein intraselläre Hypophysenprozesse teilweise schon erfassen. Supraselläres Wachstum führt durch Druck auf das Chiasma opticum zu Gesichtsfeldausfällen bis hin zum Vollbild der bitemporalen Hemianopsie, der sogenannten «Scheuklappen-Blindheit». Von den lokalen Komplikationen des Hypophysenprozesses ist neben dem Sinus-cavernosus-Syndrom mit Doppeltsehen als Folge von Oculomotorius- und Abducens-Parese die akute Blutung in die Нypophyse, die mit schweren retrobulbären und Schläfenkopfschmerzen einhergeht, am meisten gefürchtet. Sie hat eine Mortalität von bis zu $50 \%$, da es neben der gelegentlich vollständigen Zerstörung des Wachstumshormonproduzierenden Gewebes («Autohypophysektomie») auch zu einem Ausfall sämtlicher Partialfunktionen des Hypophysenvorderlappens bis hin zum 
klinischen Bild eines hypophysären Komas kommen kann. Das Ganze kann ein meningitisches Bild vortäuschen. Auch im Spätstadium kann es durch mechanische Alteration und Zerstörung des normalen Hypophysengewebes zu einer gestörten Sekretion der übrigen Hypophysenvorderlappenhormone mit sekundärem Hypogonadismus, sekundärer Hypothyreose und zuletzt sehr selten - sekundärer Nebennierenrindeninsuffizienz kommen.

Als Folge des hohen Insulin-antagonistischen und damit diabetogenen Wachstumshormonspiegels kommt es durch die permanente Forderung zu einem sekundären Hyperinsulinismus. Fast $2 / 3$ der akromegalen Patienten zeigen eine gestörte Kohlenhydratstoffwechsellage und nahezu 1/4 einen manifesten Diabetes mellitus, der bei der Hälfte der Patienten bereits vor der Diagnose der Akromegalie bekannt ist. Eine erhöhte basale Prolaktinsekretion wird bei $20-30 \%$ aller Patienten beobachtet, führt jedoch nur in wenigen Fällen zu einer klinisch nachweisbaren Galaktorrhoe.

Die mittlere Lebenserwartung der akromegalen Patienten wird bei unbehandelter Krankheit mit etwa 13 Jahren angegeben. Die Todesursachen sind mannigfaltig: Hirndruck, Blutungen in den Tumor und das Schädelinnere, Komplikationen der Zuckerkrankheit und Funktionsstörungen des übergroßen Herzens spielen dabei die wesentlichste Rolle.

\section{Psychische Veränderungen bei akromegalen Patienten}

Die Akromegalie führt mit ziemlicher Regelmäßigkeit zu einer Wesensänderung ${ }^{1}$ :

1. Die Angetriebenheit kann vermehrt oder vermindert sein. Extreme in der Veränderung der Angetriebenheit sind maniforme Zustände einerseits und schwere apathische Zustände andererseits.

2. Die Veränderungen der inneren Gestimmtheit sind naturgemäß mit den Veränderungen der Angetriebenheit aufs engste verbunden. Häufig ist eine Verstimmung gleichzeitig gekennzeichnet durch stumpfe Apathie und zufriedene Heiterkeit. (Sie wurde schon 1912 durch Frankl-Hochwart «Hypophysärstimmung» genannt, doch kommt sie bei vielen endokrin Kranken, nicht nur bei Hypophysenkranken vor.) Die Veränderungen der Antriebe und der Stimmungen können dem eigenen Wesen fremd erscheinen. Depersonalisationserlebnisse können vorkommen.

3. Auch die Veränderungen der elementaren Triebe endokrin Kranker bestehen kaum in Triebperversionen: Ihre Triebe sind übersteigert oder abgeschwächt. Besonders auffällig sind Veränderungen in Durst und Hun- 
ger, im Bedürfnis Wärme zu suchen oder sie zu meiden, im Bedürfnis, sich zu bewegen und zu wandern oder die Ruhe zu pflegen und sich im Heim zu verkriechen, im aggressiven Bedürfnis, sich mit andern zu messen, in einer auf die rein körperliche Lustgewinnung gerichteten Sexualität, in einer ganz primitiven Teilkomponente menschlicher Mütterlichkeit, einem Trieb, Kleines zu hätscheln und zu umsorgen.

Bei allen diesen Veränderungen der Antriebe, Stimmungen und Triebe endokrin Kranker fällt die Unberechenbarkeit ihres Auftretens und Zurücktretens auf. Ganz unvermittelt können endokrin Kranke z. B. von Heißhunger oder sexueller Gier gepackt sein, um ebenso unvermittelt jedes Interesse am Essen oder an sexueller Aktivität zu verlieren. Ganz generell ist die Phasenhaftigkeit im psychischen Leben verändert.

Ein Akromegaler, der zufolge seines Hypophysenadenoms zu erblinden drohte, erklärte in einer satten und gleichgültigen Stimmung, das sei ihm gleich, er bemühe sich deshalb noch lange nicht, den Arzt aufzusuchen ${ }^{19}$.

\section{Geschichte der Akromegalieforschung}

Die ersten Beschreibungen von Gigantismus und Akromegalie findet man am Ende des 18. Jahrhunderts. Die medizinische Forschung hat hier oft abenteuerliche Wege eingeschlagen. Beim ersten gut dokumentierten Fall handelt es sich um Charles O'Brian, einen damals bekannten irischen Riesen, der mit einer Körpergröße von fast 2,5 m in England Aufsehen erregte. John Hunter, der damals wohl berühmteste Anatom und Chirurg Englands, dem wir fundamentale anatomische und pathologische Studien verdanken, wollte das Skelett des Riesen in seine anatomische Sammlung aufnehmen. Als Hunter hörte, daß sich der Gesundheitszustand des damals 22jährigen O'Brian verschlechterte, beauftragte er seinen Mitarbeiter Howison, dem Patienten zu folgen, um sofort vom Tod berichten zu können. Dies kam O'Brian zu Gehör. Da er sich nicht autopsieren lassen wollte, verfaßte er ein Testament, in dem er verfügte, daß seine Leiche streng bewacht und dann sobald wie möglich in einem Bleisarg im Meer versenkt werden müsse. Nachdem der Patient verstorben war, soll sich, nach der lokalen Presse, eine ganze Schar von Chirurgen um das Trauerhaus versammelt haben. Der Sarg wurde am 18. Juni 1783 versenkt. Einige Leichenjäger hätten sich, nach einem andern Bericht, Taucherausrüstungen besorgt, um den Sarg später wieder zu bergen. 
Alle hatten aber die Rechnung ohne John Hunter gemacht. Er hatte mit einem damals phantastischen Betrag von $500 £$ die Totengräber bestochen. Die Leiche wurde bei Nacht und Nebel aus dem Trauerhaus gestohlen und in einem Pferdefuhrwerk, das aus Sicherheitsgründen unterwegs sogar gewechselt wurde, ins Huntersche Labor gebracht. Aus Angst vor einer Entdeckung wollte Hunter keine Zeit mit der üblichen Methode der Präparierung des Skelettes verlieren. Deswegen wurde, so berichtet wenigstens der Biograph Paget, der Körper in Stücke geschnitten und das Fleisch durch Kochen vom Skelette getrennt. Daher rühre die auch heute noch sichtbare braune Verfärbung des Präparates, das immer noch im Royal College of Surgeons in London aufbewahrt wird. Der Schädel wurde erst etwa 1909 von Harvey Cushing und Sir Arthur Keith genauer untersucht, die nachweisen konnten, daß O'Brian an einem Hypophysentumor gelitten hatte. Cushing bemerkte, daß hier Hunters Sammeltrieb über den Wissensdurst gesiegt und ihn um den Ruhm der Erstbeschreibung des Akromegalie-Gigantismus-Syndroms gebracht habe.

In den folgenden Jahren bis zur endgültigen Benennung der Krankheit durch Pierre Marie wurden immer wieder vereinzelte weitere Fälle mitgeteilt, wobei der Patient, der von Fritzsche und Klebs beobachtet wurde, wohl am besten dokumentiert ist.

Anfangs September 1882 wurde der Elmer Gemeindeweibel Peter Rhyner in die «Kantonale Krankenanstalt» in Glarus, wie sie damals noch hieß, eingeliefert. Der 1838 geborene Rhyner von Elm im Kt. Glarus, des Oswald und der Anna geb. Zentner, litt an einer höchst sonderbaren Krankheit, die sich erstmals im Alter von 36 Jahren bemerkbar machte (Abb. 4). Sie begann in den Händen mit spannenden und zerrenden Schmerzen, denen Schwellungen folgten, die sich nicht wegdrücken ließen, also kein Ödem waren. Allmählich stiegen die Schmerzen in den Armen aufwärts und zeigten sich bald im ganzen Körper, mit Ausnahme der Beine, die erst in den letzten 2 Jahren zu schmerzen begannen. Mit der Zeit ging auch eine äußerliche Veränderung vor sich. Zuerst fiel Peter Rhyner eine Verdickung der Finger auf, und schließlich war die Vergrößerung, das Wachstum der Hände und Füße, der Knie, der Ohren und Lippen, der Zunge, des Halses, ja des ganzen Kopfes nicht mehr zu übersehen. Die abnorm verdickte Haut ließ sich in großen, weichen Falten abheben. Zum Krankheitsbild gehört aber auch eine abnorme Verformung der Knochen und Knorpel des Skelettes, und als bei Rhyner die Wirbelsäule sich allmählich verkrümmte, wurde dies der schweren Arbeit zugeschrieben. Geschlechtliche Regungen, die früher vor- 


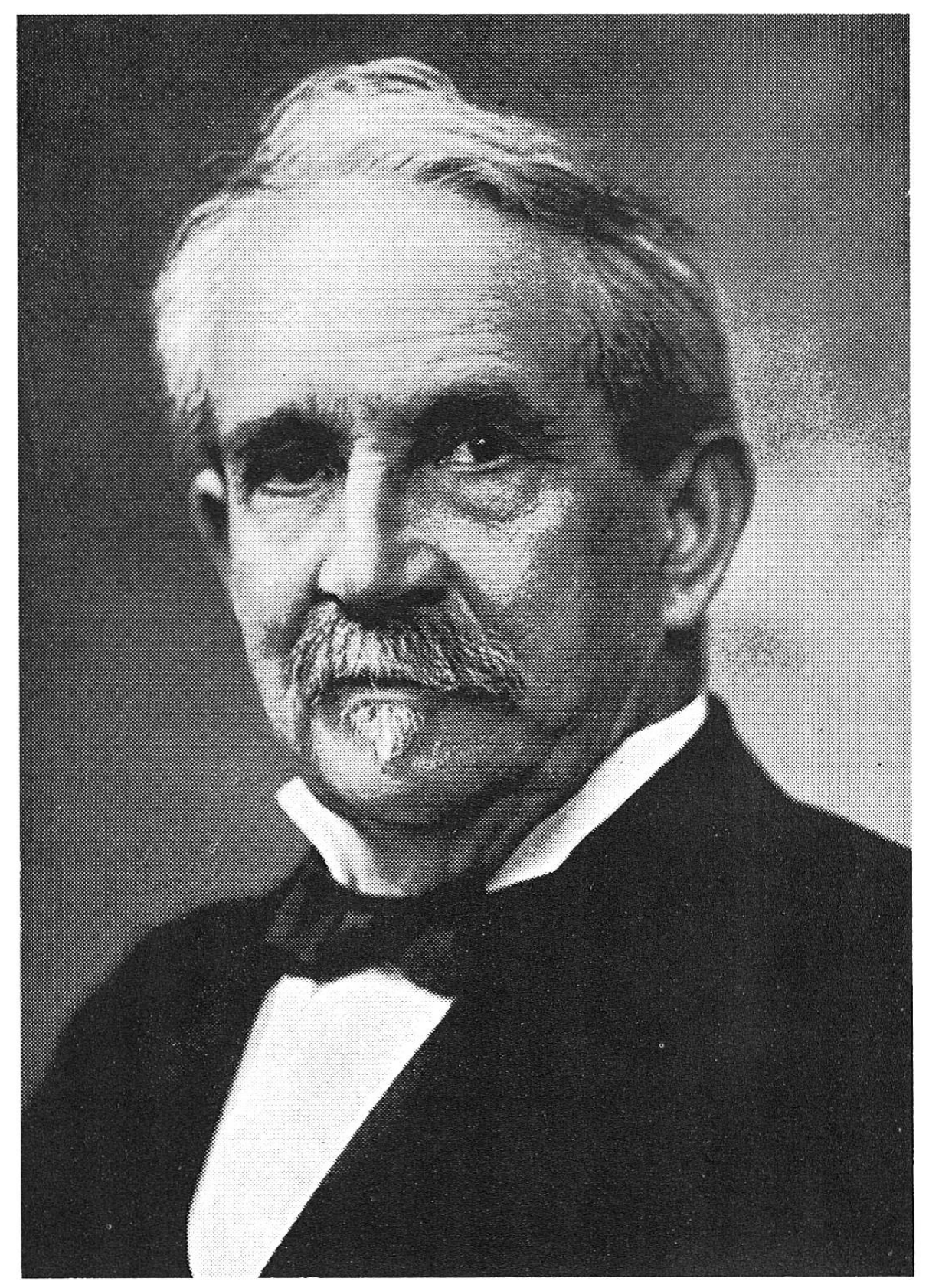

Abb. 1. Der erste Glarner Spitalarzt Dr.med. Christian Friedrich Fritzsche (1851-1938) Foto: H.Schönwetter-Elmer

handen waren, fehlten seit vielen Jahren. Der Patient will eine Abnahme des Gedächtnisses bemerkt haben, was aber objektiv im Gespräch nicht aufgefallen war. Die Augen waren schwächer geworden, das Gehör aber und die übrigen Sinne waren gut erhalten. Peter Rhyner soll sogar über einen ganz gesunden Mutterwitz verfügt haben! Am bedenklichsten wirkte sich eine 


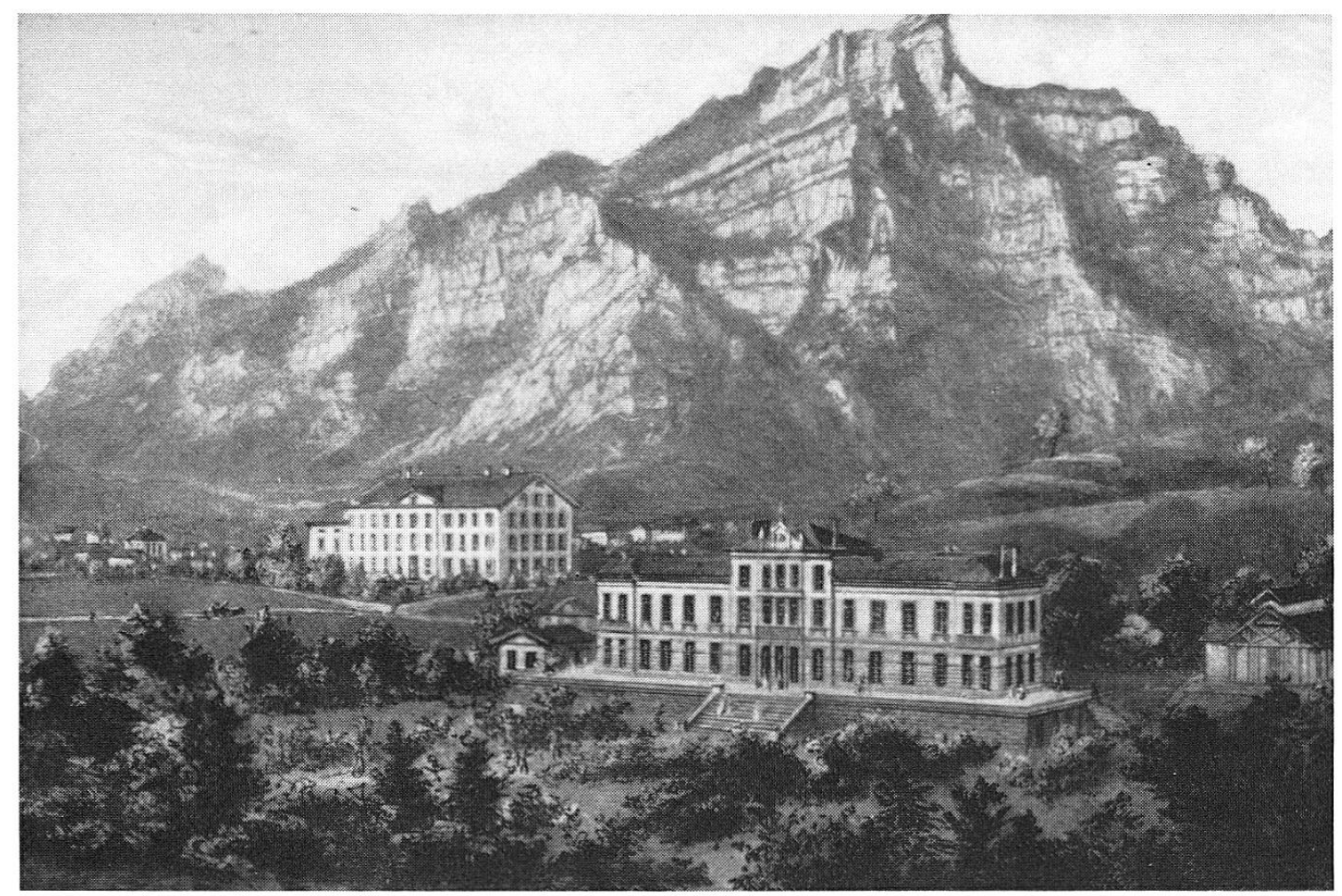

Abb.2. Glarus, Kantonsspital, Stahlstich von Heinrich Zollinger, 1880

zunehmende Schwäche aus, welche dem Senn die Beschäftigung auf der Alp und dem Gemeindeweibel die Ausübung seines Amtes unmöglich machte. In der letzten Zeit vermochte er kaum 5 Min. weit zu gehen, ohne mehrmals auszuruhen. Der offensichtlich schwer kranke und von seinem Leiden gezeichnete Peter Rhyner erhoffte sich wohl von einer Behandlung im Kantonsspital Glarus (Abb.2), welches im Jahre 1881 eröffnet wurde, eine Linderung seines Leidens.

Der erste Chefarzt des Kantonsspitals Glarus, Dr. med. Christian Friedrich Fritzsche (1851-1938) (Abb.1), Chirurg, Internist, Geburtshelfer und praktischer Arzt in einer Person (er war auch der Hausarzt meiner Großeltern in Glarus), nahm den Status des Patienten auf. Der merkwürdige, in keinem Lehrbuch verzeichnete Befund veranlaßte ihn, den Kranken einige Tage später in der medizinisch-zoologischen Sitzung der Vereinigung der Schweizer Naturforscher in Linthal/Kt. Glarus vorzuführen. Diese Vereinigung hielt vom 11.bis 14.September $1882 \mathrm{im}$ damals berühmten Bad Stachelberg in Linthal ihre 65.Jahresversammlung ab, präsidiert vom Badearzt Dr. F. König (Abb. 3). 
In 5 Fachbereichen wurden von Wissenschaftern Vorträge gehalten.

Nun zitiere ich den Berichterstatter der damaligen Glarner Zeitung, der «Freie Glarner»:

«Von allgemeinerem Interesse war die Vorführung eines ungewöhnlich gestalteten Mannes durch Herrn Spitalarzt Dr. Fritzsche. Es war ein Elmer Bauer von 44 Jahren. Bis in sein 36. Jahr lag dieser große, regelmäßig gebaute Mann der landesüblichen Feldarbeit ob. Da befiel ihn ein krankhaftes Riesenwachstum der obern Körperhälfte, während die untere eher zusammenschwindet. Die Knochen der Kinnladen, der Nase, der Schultern, der Arme und Hände sind ganz riesenhaft groß geworden, so daß der Mann bei vollem Bewußtsein eine hülfsbedürftige Mißgestalt vorstellt. Die anwesenden Physiologen Virchow aus Berlin und His aus Leipzig kannten höchstens 3-4 Fälle ähnlicher Mißbildung.»

Der Basler Wilhelm His, damals Professor der Anatomie in Leipzig, ein Schüler Virchows wie auch der Arzt und der erste schweizerische Fabrikinspektor Dr. Fridolin Schuler von Mollis/Kt. Glarus, der in seinen «Erinnerungen eines Siebzigjährigen» schrieb, daß Virchow seine Schüler nicht nur zum Lernen des bereits Bekannten, sondern auch zum eigenen Forschen anregte. Virchow sei in Würzburg derjenige Lehrer gewesen, der sein ganzes medizinisches Denken vorwiegend beeinflußt habe. Die Anwesenheit des Pathologen Rudolf Virchow verdankte die Gesellschaft ihrem Mitglied Wilhelm His, von dem Virchow in einer Tischrede sagte, er habe mit seinem Freunde His Freud und Leid geteilt und sei ihm deshalb auch zur Jahresversammlung nach Linthal gefolgt, an der Virchow einen Beitrag leistete mit Erklärungen über einen eiszeitlichen menschlichen Unterkiefer.

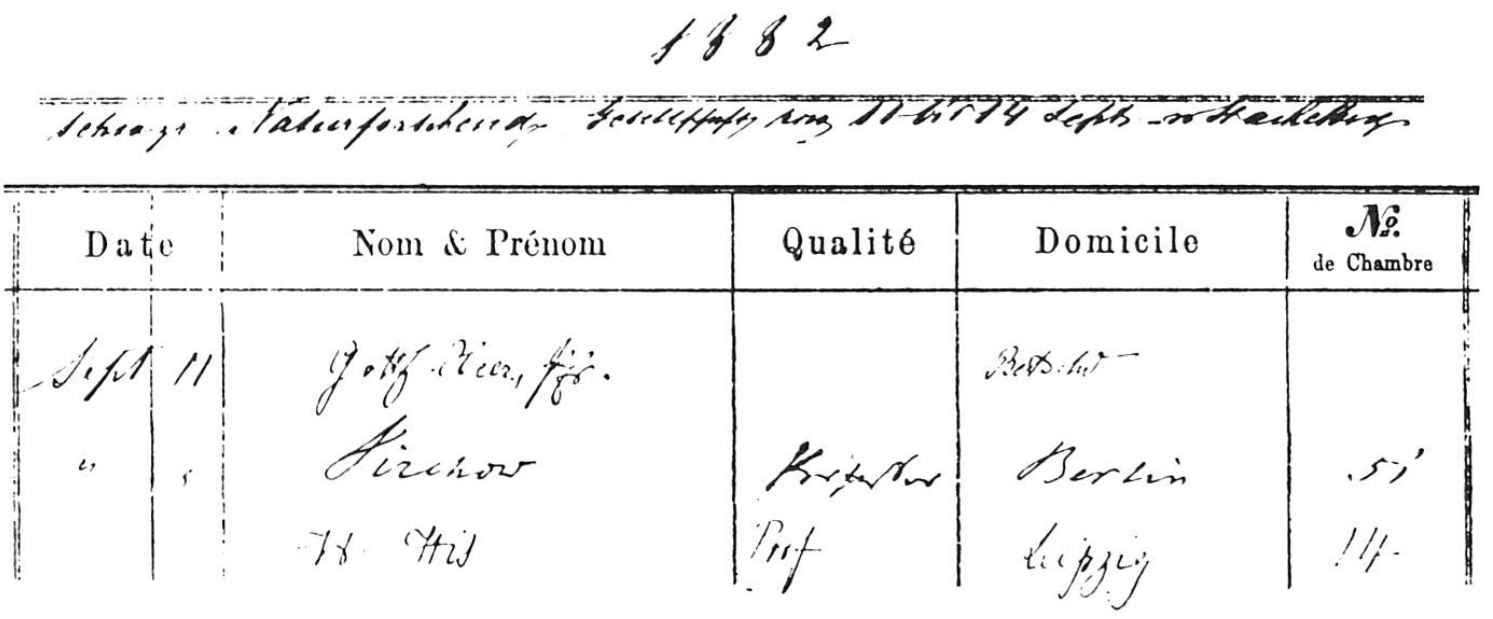

Abb.3. Die handschriftlichen Eintragungen von Pfr. Gottfried Heer, Prof. Virchow und Prof. His im Gästebuch des Kurhauses Stachelberg, anläßlich des Kongresses der Schweizerischen Naturforschenden Gesellschaft vom 11. bis 14.September 1882 
Fünf Tage nach der Sitzung im Bad Stachelberg, am 19. September 1882, starb Peter Rhyner im Glarner Kantonsspital und wurde in Glarus beerdigt.

Edwin Klebs (1834-1913), damals Ordinarius für Pathologie an der Universität Zürich, führte die Autopsie durch und fand einen Hypophysentumor, den er richtig als Adenom, d.h. als drüsenartige Wucherung, bezeichnete. Er erkannte als erster, daß diese Hypophysenvergrößerung charakteristisch für das Leiden war, da schon bei andern Fällen ähnliche Befunde mitgeteilt worden waren. Seine theoretischen Erörterungen führten aber leider zum unrichtigen Schluß, daß die Hypophysenvergrößerung Folge und nicht, wie er auch in Erwägung zog, Ursache des Leidens war.

Wie mir Dr.med.Robert Fritzsche (1891-1978), der jüngste Sohn des ersten Glarner Spitalarztes, vor 10 Jahren in Glarus mündlich mitteilte, habe Prof.Klebs bei Peter Rhyner leider der persistierenden großen Thymusdrüse mehr Beachtung geschenkt als der vergrößerten Нypophyse.

Der sorgfältig präparierte Schädel Rhyners konnte lange Zeit im Pathologischen Institut der Universität Zürich besichtigt werden.

Eingedenk der dringenden Bitte Virchows, Fritzsche möchte die Krankengeschichte Rhyners veröffentlichen, gaben Fritzsche und Klebs im Jahre 1884 eine Broschüre heraus: «Ein Beitrag zur Pathologie des Riesenwuchses», in der Krankengeschichte und Sektionsbefund beschrieben waren. Peter Rhyner bildete übrigens für Dr. Fritzsche nicht einen Einzelfall. 1886 wurde ihm eine Frau aus Schänis/Kt. St. Gallen zugewiesen, deren Leiden in verschiedenen Punkten mit demjenigen Rhyners übereinstimmte.

Diese erste exakte Beschreibung eines neuen Krankheitsbildes wurde jedoch kaum beachtet. Sie wurde nicht einmal im damals sehr verbreiteten «Jahresbericht über die Leistungen und Fortschritte in der gesamten Medicin» erwähnt. Vielleicht lag dies daran, daß Fritzsche darauf verzichtet hatte, der Krankheit einen besonderen Namen zu geben, und daß Klebs, der in der stark vergrößerten Sella turcica keine Besonderheit sah, den Fall in die große Gruppe des allgemeinen Riesenwuchses eingereiht hatte. Dabei hatte schon der Wiener Anatom Karl Langer (1819-1887), auf dessen Monographie «Wachstum des menschlichen Skelettes mit Bezug auf den Riesen» aus dem Jahre 1872 sich Klebs stützte, darauf hingewiesen, daß eine Vergrößerung der Sattelgrube nur bei solchen Riesen vorkommt, die einen «monstruösen» Unterkiefer, wulstige Lippen und vergrößerte Nasenflügel aufweisen. 
1886 gab Pierre Marie (1853-1940) (Abb.5) aus der Charcotschen Klinik in Paris ein vollständiges klinisches Bild jener Krankheit, die hauptsächlich durch eine übermäßige Entwicklung gewisser Teile des Körpers, in erster Linie des Schädels, der Gliedmaßen, des Skelettes, charakterisiert ist. Diese «entité morbide spécial» die er als «Akromegalie» bezeichnete ist begleitet von schweren Veränderungen der Hypophyse.

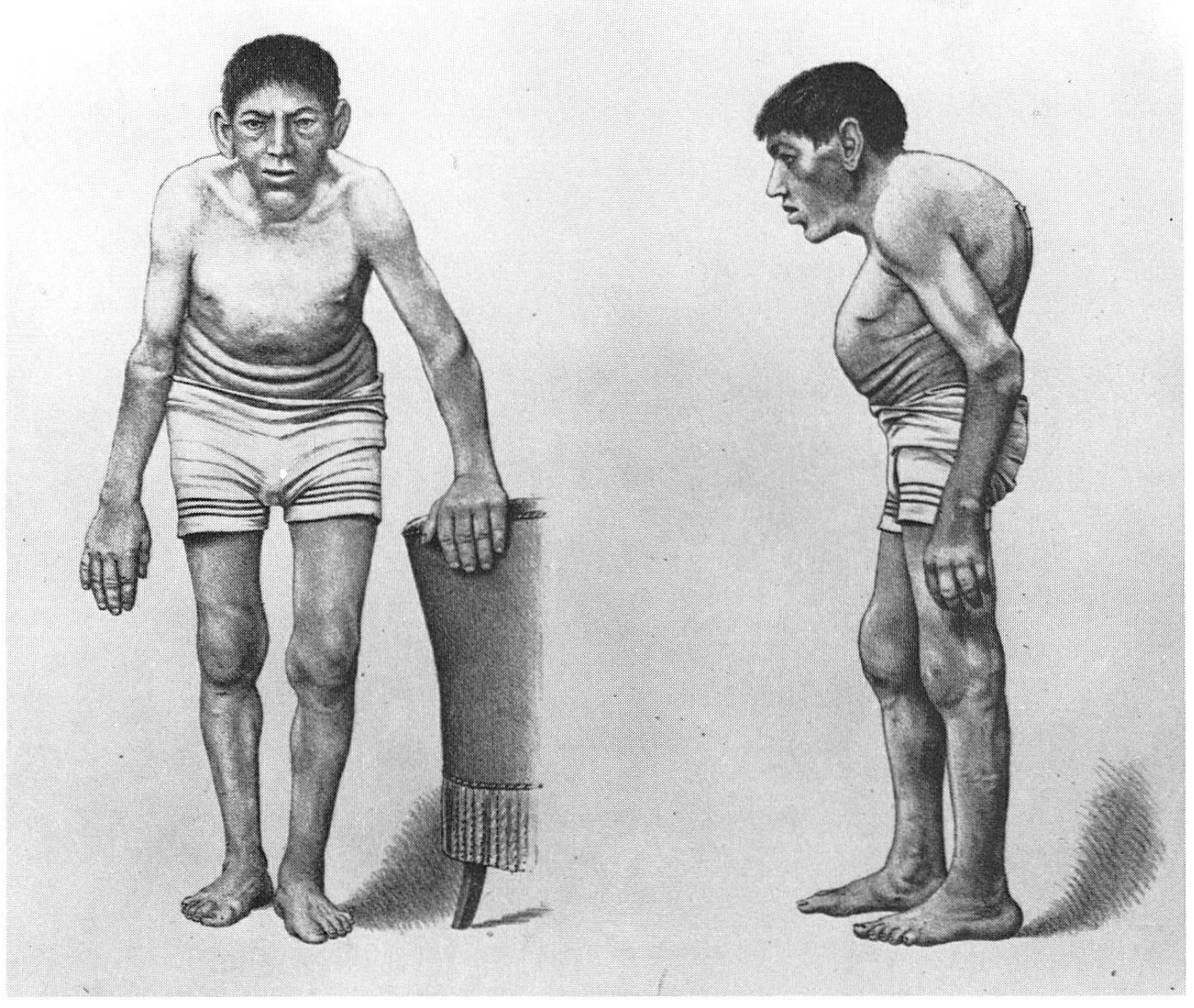

Abb.4. Der von Christian Friedrich Fritzsche und Edwin Klebs (1834-1913) beschriebene Fall von Akromegalie. Lithographie aus ihrer Arbeit «Ein Beitrag zur Pathologie des Riesenwuchses», Leipzig 1884

Heute wird die Krankheit in Frankreich vielfach als «Maladie de Marie» bezeichnet. Ursprünglich hatte Pierre Marie die Krankheit Akomakrie nennen wollen, wählte dann aber des Wohlklanges wegen die Bezeichnung Akromegalie. 


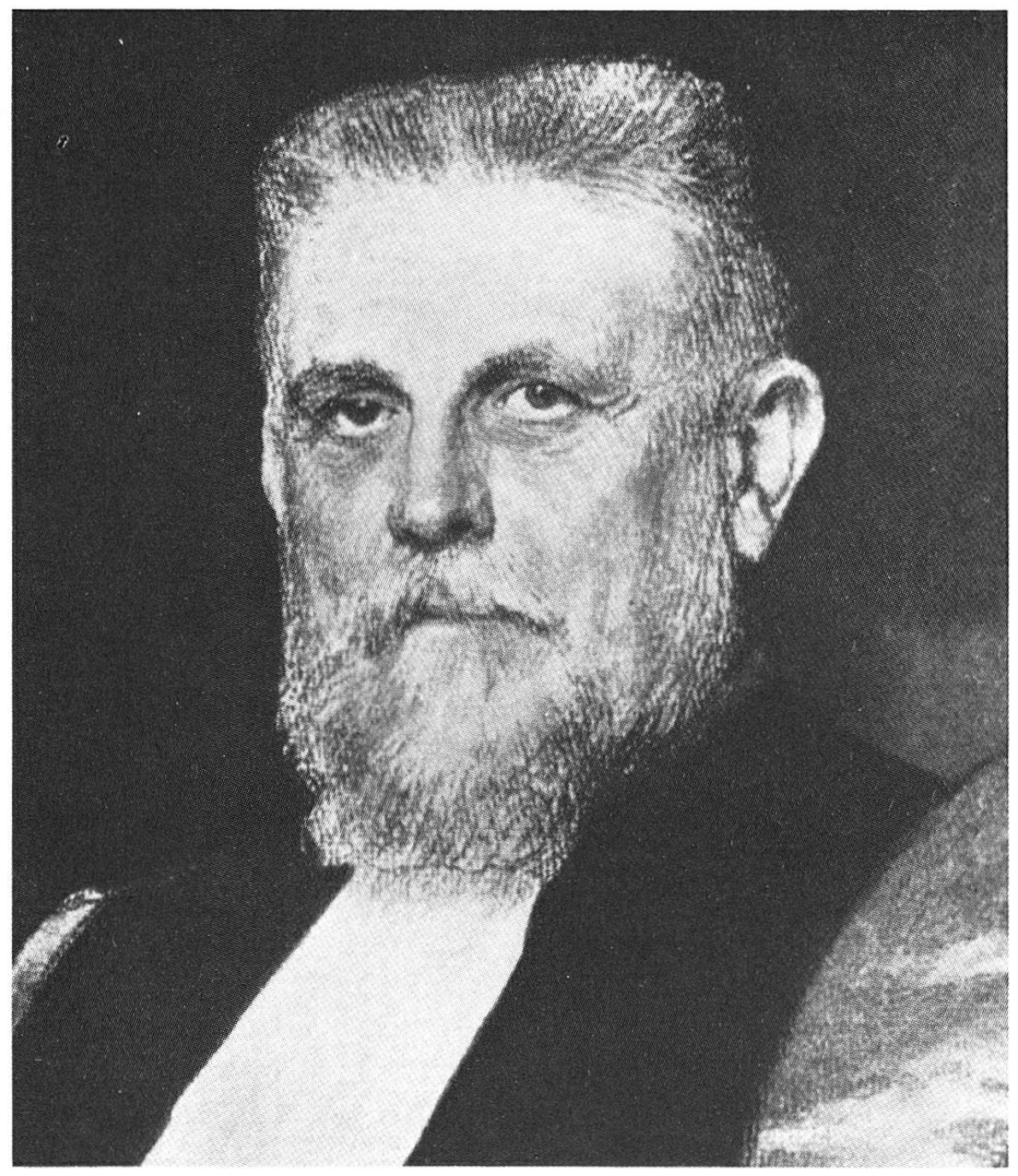

Abb.5. Der französische Neurologe Pierre Marie (1853-1940)

« $1^{\circ} \mathrm{Il}$ existe une affection caractérisée surtout par une hypertrophie des pieds, des mains et $\mathrm{du}$ visage, que nous proposons d'appeler acromégalie, c'est-à-dire hypertrophie des extrémités (non pas qu'en réalité, les extrémités soient seules atteintes pendant toute la durée de la maladie, mais parce que leur augmentation de volume est une phénomène initial et constitue le trait le plus caractéristique de cette affection.

$2^{\circ} \mathrm{L}$ 'acromégalie est tout à fait distincte du myxoedème et de la maladie de Paget (ostéite déformante), ainsi que de la leontiasis ossea de Virchow.» ${ }^{2}$

Hätte Peter Rhyner heute mit Erfolg behandelt werden können? Ja! Wir wissen jetzt, daß die Akromegalie ihren Ursprung eben in dem Tumor der Hirnanhangsdrüse hat, der abnorm gesteigerte Mengen von Wachstumshormonen produziert. Tritt die Erkrankung bereits vor der Pubertät auf, d.h. 
vor Schluß der für das Längenwachstum verantwortlichen Wachstumszone (Epiphysenfugen) im Skelettbereich, so erfolgt «nur» ein abnormer Riesenwuchs oder Gigantismus. Wahrscheinlich gehörte der Riese Melchior Thut (1736-1784) aus Linthal/Kt.Glarus, dazu, dessen Lebensgeschichte im Glarner Neujahrsboten 1967 geschildert wurde und die 1977 auch das Thema der Ausstellung im «Dorfmuseum Linthal» bildete.

Es ist interessant zu erfahren, was man sich in Elm selber über den unglücklichen einstigen Gemeindeweibel erzählt, der doch in jedem Haus ein bekannter Mann gewesen sein mußte. Lehrer Heinrich Stüssi aus Linthal erkundigte sich bei dem inzwischen verstorbenen Elmer Gemeindeschreiber Kaspar Hefti wie auch bei andern Gewährsleuten und fragte in der Verwandtschaft Peter Rhyners herum, aber man wußte nicht einmal etwas von seiner Existenz, geschweige von dessen Schicksal. Vergessen, ausgelöscht in der Erinnerung von dem furchtbaren Geschehen durch den Bergsturz vom 11. September 1881 in Elm, vor dem die Heimsuchung eines einzelnen belanglos wurde.

Sowohl Fritzsche und Klebs als auch Pierre Marie, dem übrigens die Arbeit der Erstgenannten nicht bekannt war, hatten aus der Literatur Fälle zusammengestellt, die nachträglich einwandfrei als Akromegalie-Fälle bezeichnet werden dürfen. Der früheste von ihnen ist wohl der von dem französischen Wundarzt Nicolas Saucerrotte (1741-1814) im Jahre 1772 beobachtete «Sieur Mirbeck».

Andrea Verga (1811-1895) veröffentlichte 1864 das erste vollständige Obduktionsprotokoll eines Akromegaliefalles. Als besondere Merkwürdigkeit erwähnte er, daß anstelle der Hypophyse ein großer Tumor vorhanden war, der die Sella turcica vergrößert, das Keilbein eingedrückt und die Sehnerven verschoben hatte. Verga wählte für seinen Fall die Bezeichnung «Prosopektasie». Ebenfalls bemerkenswert ist eine Mitteilung des Italieners Vincenzo Brigidi (1838-1908), der 1877 den Schauspieler Ghirlenzoni sezierte und auf die Ähnlichkeit des Skelettes mit dem eines Orang Utan aufmerksam machte (Abb.6). Das infolge seiner Krankheit groteske Aussehen Ghirlenzonis hat zu seinen Erfolgen als Mephistopheles in Goethes Faust beigetragen. Brigidi hat bei dieser Sektion als erster einen Hypophysentumor mikroskopisch untersucht.

Es ist wahrscheinlich, daß mancher in der älteren Literatur beschriebene Riese nichts anderes als ein Akromegaler gewesen ist. Dies trifft z. B. sicher zu auf einen Riesen aus dem Hofstaate des Kurfürsten und Pfalzgrafen Friedrich II. (1533-1556), dessen Bild im Jahre 1553 vom Hofmaler des 


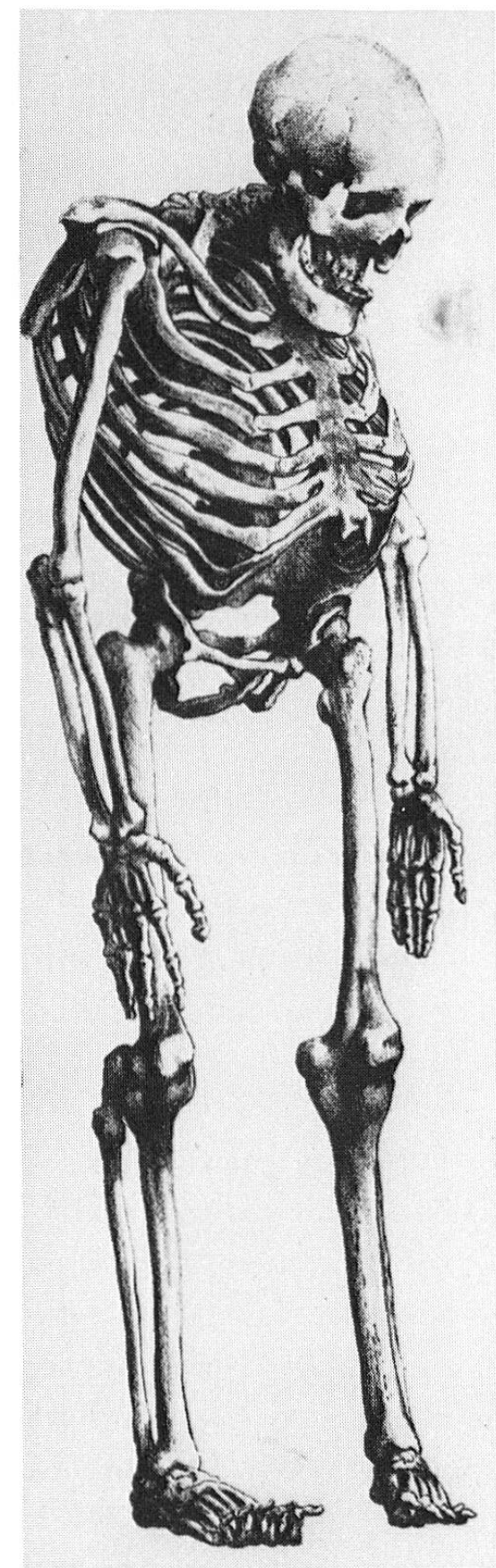

Abb.6. Skelett des akromegalen Schauspielers Ghirlenzoni. Lithographie (Ausschnitt) aus «Studi anatomo-patologici sopra un uomo divenuto stranamente deforme per cronica infermità» von Vincenzo Brigidi (1838-1908). Archivio della Scuola d'Anatomia patologica di Firenze I, 63, 1881

Kurfürsten in Lebensgröße gemalt worden ist und das noch heute auf Schloß Ambras b/Innsbruck im Tirol aufbewahrt wird.

Der Wiener Internist Maximilian Sternberg (1838-1934) weist darauf hin, daß wohl auch eine von Johann Weier (1515-1588), einem Vorläufer der Psychiatrie und Bekämpfer des Hexenwahns, im Jahre 1567 beschriebene 25jährige Riesin an Akromegalie litt, wie die Worte »Toto corpore torpida» und die Bemerkung, daß die Frau nicht menstruierte, zu beweisen scheinen. 
Möglicherweise ist das Vorbild für die Gesichtszüge des romanischen Typus des Hanswurstes, des buckligen «Pulcinella» oder «Polichinelle», ein Akromegaler gewesen. Dasselbe dürfte für die englische Figur des «Punch» zutreffen.

Die Untersuchungen von Pierre Marie, der übrigens noch keine Ahnung von der Ursache dieser Krankheit hatte, «nous n'avons encore aucune donnée certaine sur la nature de cette maladie; celle-ci cependant nous paraît bien présenter tous les caractères nécessaires pour constituer une véritable entité morbide ${ }^{2}$, regten in beträchtlichem Maße das Studium der Pathologie der Hypophyse an. In wenigen Jahren hat sich eine ganze Spezialliteratur entwickelt, welche genaue klinische und pathologische Beobachtungen über die Akromegalie und die dabei befallenen Organe enthält. Indessen vermochten diese Beobachtungen, obschon sie bei Klinikern und Pathologen ein lebhaftes Interesse für die Physiologie der Hypophyse hervorriefen, dennoch nicht die normale Funktion des Organs klarzulegen. Die Arbeiten, die die wirkliche Bedeutung der Hypophyse feststellen sollten, gingen daher auch von den Untersuchungen über die Akromegalie nicht aus. Es waren vielmehr die Beziehungen, die zwischen Schilddrüse und Hypophyse zu bestehen scheinen, die die Aufmerksamkeit der Experimentatoren fesselten $^{3}$. José Dantas de Souza Leite, ein Schüler von Pierre Marie, der 1890 die erste Monographie über Akromegalie schrieb, konnte schon 38 sichere Fälle aufzählen. Sternberg erwähnte in seiner 1897 erschienenen monographischen Darstellung dieser Krankheit 210 Fälle, während im Jahre 1932 Frederick Richard Breeks Atkinson (1867-1939) bereits sogar 1319 Fälle zusammenstellen konnte.

Obgleich die Vergrößerung der Hypophyse schon früh als ein sehr wesentliches pathologisch-anatomisches Merkmal der Akromegalie beschrieben worden war, erkannte man die ätiologische Bedeutung dieses Befundes zunächst nicht. Der erste, der im Jahre 1887 darauf hinwies, war der geniale deutsche Internist und Experimentator Oskar Minkowski (1858-1931), zu jener Zeit noch junger Assistent bei Prof. Bernhard Naunyn in Königsberg.

Der von Minkowski beschriebene Fall ${ }^{4}$ entspricht genau dem von Marie geschilderten Krankheitsbilde: Im späteren Lebensalter beginnende und allmählich zunehmende Vergrößerung der Hände und Füße, Verbreiterung der Gesichtsknochen, Hypertrophie der Nase, der Ohren, der Lippen und des Kinns, verbunden mit Anämie, allgemeiner Muskelschwäche, Kopfschmerzen, Beeinträchtigung des Sehvermögens und des Gehörs. 
«Ebenso sicher dürfte es sein, daß der von Henrot ${ }^{21}$ - wie Marie ${ }^{2}$ überzeugend ausführt, irrtümlich - als Myxödem beschriebene Krankheitsfall zu den hier in Rede stehenden Fällen zu zählen ist. - Einen Fall, welchen Fritzsche und Klebs im Jahre 1884 publicirt haben, scheint Marie übersehen zu haben, was umso bedauerlicher ist, als derselbe wohl der am sorgfältigsten beobachtete und namentlich auch am genauesten anatomisch untersuchte Fall sein dürfte. Auch ein Fall von Brigidi, welchen Fritzsche und Klebs ausführlicher referiren, ist wohl hieher zu rechnen.

Der von Marie zur Bezeichnung des in Rede stehenden Krankheitszustandes gewählte Ausdruck «Akromegalie = das äußerste Ende ` dürfte insofern ganz geeignet sein, als in der Tat die Hypertrophie der extremsten Körperteile, die am meisten in die Augen fallen, die Erscheinung in allen oben erwähnten Fällen bildete.

Bemerkenswerth ist, daß in allen bis jetzt secirten und genauer untersuchten Fällen neben einer mehr oder weniger ausgesprochenen Hypertrophie sämmtlicher inneren Organe eine besonders auffallende Vergrößerung der Hypophysis cerebri notirt ist. In dem Falle von Henrot ${ }^{21}$ hatte dieses Organ die Größe eines kleinen Hühnereis erreicht, und auch in dem Falle von Brigidi, sowie in dem von Fritzsche und Klebs wird die enorme Vergrößerung der Hypophysis erwähnt, die zu einer mächtigen Erweiterung der Sella turcica und zu einer Compression der Nervi optici geführt hatte, welche die Ursache von Sehstörungen geworden war. Auch in unserem Falle könnte sehr wohl die zunehmende Beeinträchtigung des Sehvermögens auf eine solche Compression der Sehnerven durch die sich vergrößernde Hypophysis zurückzuführen sein. - Wie die genauere mikroskopische Untersuchung in dem Falle von Fritzsche und Klebs ergeben hatte, beruhte die Vergrößerung der Hypophysis, ebenso wie die der andern Organe, auf einer gleichmäßigen Zunahme der typischen Gewebsbestandtheile neben einer stärkeren Entwicklung der Gefäße. Klebs glaubt, diese Hyperplasie der Hypophysis nur als eine einfache Theilerscheinung der allgemeinen Steigerung des Körperwachstums betrachten zu müssen und das relativ stärkere Wachsthum dieses Organes durch die größere Proliferationsfähigkeit der Drüsen - gegenüber der Hirnsubstanz erklären zu können. Dagegen ist er, wie bereits erwähnt, geneigt, der Thymushyperplasie eine ursächliche Bedeutung für den Gesammtvorgang des Riesenwuchses beizulegen ${ }^{5}$. Diese letztere Annahme muß aber entschieden noch durch weitere Untersuchungen gestützt werden. Daß aber möglicherweise die Vergrößerung der Hypophysis für den in Rede stehenden Vorgang doch nicht ganz bedeutungslos ist, dafür spricht vielleicht der Umstand, daß - wie neuerdings noch Virchow ${ }^{6}$ betont hat - ein Parallelismus zwischen dem Gewebe dieser Drüse und demjenigen eines Organs besteht, welchem gerade in neuerer Zeit besondere Beziehungen zu trophischen Vorgängen im Organismus zugeschrieben werden, nämlich dem Gewebe der Schilddrüse.

Die wichtigsten Unterscheidungsmerkmale der Akromegalie gegenüber dem Myxödem bestehen nach Marie einmal in dem Vorhandensein der Knochenhypertrophie, die bei dem Myxödem fehlt, zweitens in dem Fehlen der für das Myxödem charakteristischen Veränderungen der Haut und drittens in der sehr ausgesprochenen Verschiedenheit der Physiognomie bei beiden Krankheiten: Das verlängerte Oval des Gesichts bei der Akromegalie kontrastirt sehr auffallend mit dem 〈Vollmondgesicht〉 bei dem Myxödem.»? 
Wie aus dem Gesagten ersichtlich ist, laufen die Fäden zum Aufspüren der Ätiologie dieser Krankheit über Fritzsche, Klebs und Pierre Marie im Jahre 1887 bei Oskar Minkowski zusammen. Minkowski, der übrigens zwei Jahre später, 1889, zusammen mit von Mering zufällig entdeckte, daß totale Pankreatektomie bei geeigneten Versuchstieren einen Diabetes mellitus erzeugt, beobachtete demnach als erster die beständige HypophysenvergröBerung bei Akromegalie.

1890 behauptete de Souza Leite, daß die Akromegalie eine allgemeine, von der Hypophysenerkrankung abhängige Dystrophie sei. Diese Theorie vermochte sich jedoch nur langsam durchzusetzen, vor allem wohl deshalb, weil immer wieder Fälle mit Hypophysentumor festgestellt werden konnten, die keine Zeichen der Akromegalie aufwiesen. Manche Autoren nahmen an, die Vergrößerung der Hypophyse sei nur als eine Teilerscheinung der Hypertrophie der Mundgebilde aufzufassen, da der Hirnanhang ja entwicklungsgeschichtlich eng mit diesen zusammenhänge ${ }^{8}$. Andere neigten zu der Ansicht, daß es sich bei der Akromegalie um eine Erkrankung aller Blutdrüsen und nicht nur der Hypophyse handle, die durch ein unbekanntes Gift verursacht werde. Schließlich wurde auch noch eine Theorie der Wachstumsanomalie aufgestellt; aus der häufigen «Tierähnlichkeit» der Akromegalie, besonders was Gesicht und Schädel anbelangt, wurde auf einen Atavismus geschlossen, einen Rückfall in den Typus des anthropoiden Affen ${ }^{9}$.

Mit Nachdruck trat 1892 Roberto Massalongo (1857-1920) von der Universität Padua für die Hypophysentheorie ein. Er führte die Akromegalie auf eine Überfunktion des Hirnanhanges zurück. 1894 unterschied Augusto Tamburini (1848-1919) zwei Phasen im Verlaufe der Erkrankung, nämlich eine der Hyperaktivität der Hypophyse, der das abnorme Knochenwachstum entspricht, und eine zweite mit Aufhören der Drüsenfunktion und anschließender Kachexie, die über kurz oder lang bei allen Akromegalen zum Tode führt. Von den Anhängern der hypophysären Genese der Akromegalie wurde häufig die Frage diskutiert, ob der Hirnanhang einen Stoff produziere, der das Wachstum der peripheren Teile direkt beeinflußt, oder ob die Drüse nicht vielmehr gewisse aus dem Stoffwechsel stammende Substanzen «neutralisiere», deren dauerndes Zirkulieren im Organismus die Wachstumsanomalien hervorrufe.

Die Beobachtungen von Carl Benda (1857-1933), der in den Jahren 1900/1901 eingehende histologische Untersuchungen der Hypophysen von Akromegalen vornahm, verhalfen der Hypophysentheorie endgültig zum Sieg. 
«Es ist nicht in Abrede zu stellen, daß das zeitweilige Interesse für die Hypophysis cerebri wesentlich durch die Beobachtungen über das Zusammentreffen von Akromegalie mit Veränderungen des genannten Organes hervorgerufen worden ist ${ }^{10} . »$

«Meine eigenen Untersuchungen wurden ebenfalls vorwiegend durch die Bearbeitung von 4 Sectionsbefunden bei Akromegalie angeregt. Sie knüpften an einige Verbesserungen der mikroskopischen Technik an, über die ich im Februar in der physiologischen Gesellschaft berichtete ${ }^{22} . »$

«Ein sagittaler Durchschnitt läßt uns am besten die den Anatomen seit langem bekannte Scheidung der Drüse in zwei völlig getrennte Lappen erkennen ${ }^{11}$. Das Verhältnis der beiden Lappen ist nicht constant. Im allgemeinen ist der hintere Lappen erheblich kleiner als der Vorderlappen.

Uns soll vorwiegend der Vorderlappen beschäftigen, der von Santorini (1681-1737) ${ }^{23}$ als Glandula pituitaria potior bezeichnete Theil der Drüse. Dieser Theil zeigt in allen seinen Eigenschaften einen unverkennbaren Drüsenbau, und es ist namentlich von Virchow seine Ähnlichkeit mit der Thyreoidea von jeher betont worden.

Ich komme nunmehr zu meinen Befunden bei Akromegalie. Ich schicke voraus, daß ich mich nicht auf die heikle Frage einlassen werde, wie weit die Befunde von Hypophysisveränderungen bei der Akromegalie ein nothwendiger oder gar ein kausaler Bestandtheil dieses Krankheitsbildes seien. Ich meine allerdings, daß wir keineswegs aus dem Fehlen der Hypophysiserkrankung bei einer Anzahl von Akromegaliefällen, wie dasselbe ja beglaubigt ist, schließen können, daß die Veränderungen der Hypophysis nur ein zufälliger Befund bei der Akromegalie sind.

Wenn man nun von solchen Stellen aus die Grenzschicht gegen das Tumorgewebe verfolgt, finden sich in jedem der vier Fälle Übergangsbilder, die deutlich das Hervorgehen der Geschwulst aus dem Drüsengewebe erweisen. Das Hauptgewicht lege ich aber darauf, da $\beta$ ich in dreien der vier Fälle fand, daß das Geschwulstgewebe fast ausschließlich aus den stark gekörnten Zellen besteht.

Ich meine darnach, daß wenigstens in meinen vier Fällen von Akromegalie der vorwiegende Character der Hypophysenveränderung als einer hyperplastischen gewahrt ist, daß diese Hyperplasie ausschließlich das hauptsächlich functionswichtige Element, die gekörnten Epitelzellen betrifft. Diesen bei der Akromegalie erhobenen Befunden stelle ich schließlich noch einen Tumor der Sella turcica ohne Akromegalie gegenüber.

Ich hoffe aber gezeigt zu haben, daß sich sowohl in der normalen wie in der pathologischen Hypophysis Flesch's chromophile Zellen, die gekörnten Zellen als dasjenige Element zu erweisen scheinen, aus dessen Verhalten sich einheitliche Anschauungen von dem normalen und pathologischen Functionszustand der Drüse gewinnen lassen ${ }^{10} . »$

Wie aus dem vorher Erwähnten hervorgeht, fand also Benda, daß stets nur der Vorderlappen verändert ist, und zwar infolge einer Vermehrung der chromophilen Zellen. Er ist also der Entdecker des eosinophilen Adenoms des Hypophysenvorderlappens, das heute allgemein als die Ursache der Akromegalie gilt. Diese neuartige Theorie, daß ein abnormes Gewebe, d.h. ein Tumor, nicht nur destruktiv wachse, sondern auch etwas produzieren könne, widersprach der damals anerkannten Lehre, die immer noch eine 
Behandlung der Krankheit mit Hypophysenextrakten empfahl. Benda hat somit die Existenz des Wachstumshormons zum voraus geahnt. Den glänzenden Beweis der Richtigkeit seiner Theorie lieferten dann erst 1921 Evans und Long, die durch Injektion von Hypophysenextrakten bei Versuchstieren einen künstlichen Riesenwuchs hervorrufen konnten.

Große Schwierigkeiten bereitete es, die Akromegalie vom gewöhnlichen Riesenwuchs abzugrenzen, vor allem deshalb, weil die Akromegalen häufig zugleich Riesen sind, und umgekehrt Riesen nicht selten akromegale Züge aufweisen. Daß diese Tatsachen für Beziehungen zwischen Akromegalie und Riesenwuchs sprachen, ist einleuchtend. In diesem Sinne schreibt Massalongo im Jahre 1892: «Akromegalie ist ein abnormer Spätriesenwuchs»; und Anfang 1895 wurden von den beiden Franzosen Edouard Brissaud (1852-1909) und Henry Meige (1866-1940) nachdrücklich vertreten: Akromegalie und Riesenwuchs sind eine und dieselbe Krankheit. Überfunktion der Hypophyse in der Jugend führt zum Riesenwuchs, nach Abschluß des Wachstums zur Akromegalie. Der Riesenwuchs ist also «die Akromegalie der Jugend». Die nicht seltene Kombination von Akromegalie und Riesenwuchs erklärten Brissaud und Meige durch ein Fortbestehen der Hypophysenerkrankung über die Pubertät hinaus.

\section{Behandlung der Akromegalie}

Solange man über die Ätiologie der Akromegalie im unklaren war, konnte die Therapie nur rein symptomatisch sein. So wurde um die Jahrhundertwende unter anderem mit Schilddrüsen- und Hypophysenpräparaten behandelt, allerdings ohne eindeutigen Erfolg.

«Die Erkrankungen (Tumoren) der Hypophyse erscheinen unter dreierlei Bildern:

1. Als Akromegalie, beschrieben zuerst von Pierre Marie im Jahre $1886^{2}$.

Sie beruht in einer Vergrößerung der Hände, der Füße, der Nase und Zunge, in Verdickung der Lippen etc. und ist regelmäßig mit Störungen in der Funktion der Geschlechtsdrüsen verbunden. Sehstörungen können vorhanden sein.

2. Als Degeneratio adiposogenitalis, ein Krankheitsbild, das zuerst Fröhlich aus dem Ambulatorium von Frankl Hochwarts im Jahre 1901 beschrieb ${ }^{12}$.

Es beruht in einer Verfettung des Organismus, regelmäßig begleitet von Störungen in der Funktion der Geschlechtsdrüsen und trophischen Störungen, z.B. Ausfall der Bart-, Achsel- und Schamhaare. Auch hier können Sehstörungen vorhanden sein.

3. Als Sehstörungen ohne auffallende Allgemeinerscheinungen. Die Sehstörungen können bis zur Erblindung fortschreiten und regelmäßig läßt sich auch eine Alteration in der Funktion der Geschlechtsdrüsen konstatieren; häufig finden sich geringe Struma. 
Kopfschmerz kann bei allen drei Krankheitsformen in verschiedener Intensität bestehen, kann aber auch ganz fehlen.

Interessant und wichtig ist, daß die Akromegalie nur durch Tumoren des Vorderlappens hervorgerufen wird, und zwar - wie Benda nachwies - nur durch Adenome oder Adenokarzinome. Es handelt sich dabei höchstwahrscheinlich um eine Hypersekretion.

Ein außerordentlich wichtiges Hilfsmittel bei der Diagnose der Hypophysentumoren ist die röntgenologische Untersuchung, worauf zuerst Oppenheim ${ }^{13}$ hinwies; sie ermöglicht, die durch den Tumor hervorgerufene Erweiterung der Sella festzustellen.

Die Therapie der Hypophysentumoren kann, wie die der anderen Tumoren, nur eine chirurgische sein. Die operativen Methoden lassen sich in intrakranielle und extrakranielle scheiden.

Der erste, der Hypophysenoperationen ausführte, war Horsley ${ }^{14}$; er operierte auf intrakraniellem Wege von der mittleren Schädelgrube aus. Doch gewannen diese Eingriffe keine Bedeutung, da darüber nur eine kurze Bemerkung, aber keine näheren Angaben, insbesondere betr. der Resultate, vorliegen.

Einen zweiten intrakraniellen Weg und zwar von der vordern Schädelgrube aus, gab Krause $^{15}$ an. Doch führte er meines Wissens auf diese Art eine Hypophysenoperation nicht aus, sondern entfernte einmal ein Projektil aus der Sellagegend, ein andermal ein Fibrosarkom in dieser Gegend, letzte Operation mit letalem Ausgang.

Praktische Bedeutung gewann die Hypophysenchirurgie, als Schloffer ${ }^{16}$ im Jahre 1907 einen Hypophysentumor auf nasalem Wege operierte.

Um die Entwicklung und Anerkennung dieser Methode machten sich besonders von Eiselsberg und Hochenegg verdient.

Hochenegg ${ }^{17}$ gelang als erstem, eine Patientin mit Akromegalie erfolgreich zu operieren und zu zeigen, daß durch die Hypophysenoperation die akromegalischen Symptome sich rückbilden. Seither wurde die Operation von verschiedenen Chirurgen in dieser oder modifizierten Form bis zum heutigen Tage 53mal ausgeführt, 32mal mit Erfolg, 21mal mit Exitus letalis. Die Mortalität beträgt somit 39,6 pCt.

Ich habe somit bis zum heutigen Tage 12 Operationen wegen Hypophysentumor ausgeführt, 10mal mit Erfolg, zweimal mit Exitus letalis.

In nahezu allen Fällen konnten die Krankheitssymptome (Sehstörungen, Akromegalie, Kopfschmerzen) durch die Operation bedeutend gebessert werden.

Schon nach dem bisherigen Ergebnis halte ich mich für berechtigt, zu erklären, daß die Rhinologie in der endonasalen Hypophysenoperation ein neues, ebenso interessantes als wichtiges Arbeitsgebiet gewonnen hat und daß es nun Sache der Fachkollegen ist, durch Wiederholung dieser Operation in geeigneten Fällen den neugewonnenen Besitz zu einem dauernden zu gestalten ${ }^{18}$.»

Die Anfangs hohe Operationsmortalität von $35 \%$ konnte im Laufe der Zeit vor allem durch eine ständig verfeinerte Operationstechnik allmählich auf $10 \%$ und weniger gesenkt werden. Besondere Verdienste erwarb sich dabei der große amerikanische Hirnchirurg Harvey Cushing (1869-1939).

\footnotetext{
Als ein sehr wertvolles diagnostisches Hilfsmittel hat sich die Röntgen-
} 
diagnostik erwiesen, denn sie ermöglicht, durch die Feststellung einer vergrößerten Sella turcica indirekt das Vorhandensein einer Hypophysengeschwulst nachzuweisen. Wie bereits erwähnt, wies im Jahre 1899 Hermann Oppenheim ${ }^{13}$ auf diese diagnostische Möglichkeit hin und zeigte dabei das erste derartige Röntgenbild. 1902 demonstrierte Antoine-Louis-Gustave Béclère (1856-1939) in einer Sitzung der «Société médicale des Hôpitaux de Paris», mehrere Röntgenaufnahmen von Patienten mit Akromegalie, agromegalem Gigantismus und Gigantismus mit Vergrößerung des Türkensattels und betonte nachdrücklich die Bedeutung der Röntgenographie für die Diagnose der Akromegalie.

Über die Behandlung der Akromegalie durch Röntgenbestrahlung der Hypophyse berichteten zu Beginn des Jahres 1909 im Abstand von wenigen Wochen Alberto Gramegna in Turin und Béclère in Paris. Béclère äußerte sich sehr positiv über diese Therapie, welche heute noch als eine Methode der Wahl gilt.

Hauptziel der Therapie ist die weitgehende Normalisierung des erhöhten Wachstumhormonspiegels, um Spätkomplikationen zu vermeiden. Die Behandlung der Akromegalie ist auch heute noch ein schwieriges therapeutisches Problem, und zwar nicht nur, weil oft die Einsicht des Patienten zu therapeutischen Maßnahmen fehlt - die Akromegalie tut ja primär nicht weh -, sondern weil es mit keiner der bestehenden Möglichkeiten immer zuverläßig und risikolos gelingt, einen Stillstand und eine Rückbildung der Weichteil- und Stoffwechselveränderungen zu erzielen, ohne dabei das Risiko und die Nachteile (z. B. Kinderlosigkeit) einer Ausschaltung anderer Regelkreise durch die ablativen neurochirurgischen oder radiologischen Techniken und damit unter Umständen lebenslange Substitution in Kauf zu nehmen. Die Erfolge aller Methoden, insbesondere der neurochirurgischen Techniken, sind umso größer und die Komplikationen umso geringer, je früher der akromegale Patient einer Therapie zugeführt wird; keine der aufgeführten Therapieformen hat eine größere Mortalität als die unbehandelte Akromegalie. Die lokalen Komplikationen des Hypophysenprozesses sind absolute Indikation für ein neurochirurgisches Vorgehen. Neben den neurochirurgischen und radiologischen Techniken, die darauf abzielen, die vermehrte Ausschüttung von Wachstumshormon durch Entfernung oder Zerstörung der pathologisch produzierenden Gewebeanteile auszuschalten, besteht in den letzten Jahren in zunehmendem Maße Interesse, die vermehrte Wachstumshormonsekretion auf pharmakologischem Wege zu supprimieren. 
Nachdem der kurzfristige Suppressionseffekt von L-Dopa auf den Wachstumshormonspiegel bei einem Teil der Akromegalen entdeckt worden war, fand man 1974 auf der Suche nach andern dopaminergen Pharmaka mit protrahierter Wirkung den Wachstumshormon-supprimierenden Effekt des semi-synthetischen Ergotalkaloids Bromocriptin, dessen Prolaktin-supprimierender Effekt seit längerem bekannt und bereits klinisch erprobt war. Unter einer Langzeitbehandlung mit langsam einschleichender Dosierung auf 15-30 mg/die, in seltenen Fällen bis zu $60 \mathrm{mg} /$ die, kommt es zu einer anhaltenden Suppression der pathologisch erhöhten Wachstumshormonspiegel bei mehr als $50 \%$ der Patienten, in einigen Fällen bis in den Normbereich hinein.

Unter Bromocriptin-Dauerbehandlung bessert sich bei den meisten Patienten der subjektive und objektive klinische Befund. Ein Teil der Patienten mit weniger deutlichem Ansprechen der erhöhten Wachstumshormonspiegel berichtet über eine Besserung der subjektiven Symptomatik unter dieser Therapie. Ob eine lebenslange Bromocriptin-Behandlung bei Patienten mit Akromegalie durchführbar ist, bleibt nach erst fünfjähriger Therapie-Erfahrung abzuwarten ${ }^{20}$.

Die Hypophyse als innersekretorische Drüse und ihre Beziehung zu anderen Körperteilen wird durch zahlreiche Versuche mehr und mehr erkannt. Es ist gelungen, der Akromegalie viel von ihrem Schrecken zu nehmen.

\section{Anmerkungen}

1 M. Bleuler: Endokrinologische Psychiatrie, Stuttgart: Thieme 1954, S. 141.

${ }^{2}$ P.Marie: Sur deux cas d'acromégalie, Revue de Médecine, 6. année, Nr.4, Paris 1886, S.333.

3 E.von Cyon: Die Gefässdrüsen als regulatorische Schutzorgane des Zentral-Nervensystems, Berlin 1910, S.134.

${ }^{4}$ O. Minkowski: Über einen Fall von Akromegalie, Berliner klinische Wochenschrift, 24, 1887, S. 371-372.

J.G., ein Musiker aus Rußland, 38 Jahre alt, stammt aus gesunder Familie, in welcher niemals irgend welche seinem Leiden ähnliche Affectionen und auch keinerlei Nervenkrankheiten vorgekommen sein sollen. Patient selbst hat in seiner frühesten Kindheit die Masern und in seinem 9. Lebensjahr einen Typhus überstanden. Im Alter von 20 Jahren heirathete er und zeugte 8 Kinder, welche zwar schwächlich, aber gesund und gut entwickelt sein sollen.

Im Jahre 1877, als Patient 28 Jahre alt war, fiel es ihm auf, daß seine Finger dicker zu werden anfingen. Er sah sich genöthigt, einen Ring, welchen er getragen hatte, abzulegen, 
weil derselbe ihm zu eng wurde. Im übrigen legte Patient dieser Erscheinung keine besondere Bedeutung bei. Erst ein paar Jahre später, im Jahre 1879, wurde er auf seinen Krankheitszustand aufmerksam, als sich allmählich Kopfschmerzen einstellten, die anfangs zwar nicht heftig waren, aber beständig, Tag und Nacht anhielten und nur in ihrer Intensität bisweilen schwankten. Er bemerkte auch sehr bald, daß seine Füße größer zu werden anfingen: Die Stiefel wurden ihm zu enge, statt der Gummischuhe No.9 mußte er bald No.10, No.11 und schließlich No.12 tragen. Auch die Hände nahmen an Umfang immer mehr zu, so daß Patient bald nicht mehr Violine spielen konnte, weil er nicht im Stande war, reine Töne zu greifen. Er fing an, Cornet à piston zu blasen, und hierbei sah er sich bald genöthigt, ein größeres Mundstück zu verwenden, weil die Lippen ebenfalls dicker geworden waren. Auch an der Nase und an den Ohren constatirte er in letzter Zeit eine auffallende Verdickung; seine ganze Gesichtsform soll sich in den letzten Jahren verändert haben. Auch wurden in den letzten Jahren die Kopfschmerzen immer intensiver; zu dem beständig anhaltenden, nur mäßig starken Schmerz traten noch Anfälle von außerordentlich «krampfartigen» Schmerzen in der linken Kopfhälfte ein, welche sich anfangs seltener, später immer häufiger wiederholten. Außerdem stellte sich im Sommer 1886 auch eine Beeinträchtigung des Sehvermögens erst auf dem linken, später auch auf dem rechten Auge ein, welche sich in der letzten Zeit so gesteigert hatte, daß Patient beim Musiciren nicht mehr die Noten lesen konnte. Auch das Hörvermögen soll auf dem linken Ohre herabgesetzt sein.

${ }^{5}$ Siehe auch mündliche Mitteilung von Herrn Dr.med. Robert Fritzsche, anläßlich meines Vortrages über die Akromegalie an der Hauptversammlung der Medizinischen Gesellschaft des Kt. Glarus, anfangs Dezember 1971 in Glarus.

${ }^{6}$ R. Virchow: Über Myxoedema; Vortrag, gehalten in der Sitzung der Berliner Medicinischen Gesellschaft vom 2. Februar 1887.

Berliner klinische Wochenschrift, 1887, No.8.

7 O. Minkowski: Über einen Fall von Akromegalie, s. oben S. 374.

${ }^{8}$ Hch. Rathke: Über die Entstehung der Glandula pituitaria, S. 482.

${ }^{9}$ R.Abderhalden: CIBA Zeitschrift, die innere Sekretion, Basel 1951, S. 4575.

${ }^{10}$ C. Benda: Beiträge zur normalen und pathologischen Histologie der menschlichen Hypophysis cerebri, S. 1205-1210.

${ }^{11}$ Die Einteilung der Hypophyse in Vorder- und Hinterlappen geht auf Giovanni Domenico Santorini (1681-1737), Jacques Bénigne Winslow (1669-1760) und auf Albrecht von Haller (1708-1777) zurück, in: H.P. Schönwetter, Zur Vorgeschichte der Endokrinologie, Diss. Zürich 1968, S. 32 .

12 A. Fröhlich: Ein Fall von Tumor der Hypophysis cerebri ohne Akromegalie, Wiener klinische Rundschau 15, Nr.47, 23. November 1901, S. 883-886 und Nr. 48 S. 906-908.

${ }^{13}$ H.Oppenheim: (1858-1919) wies 1899 in einer Sitzung der «Berliner Gesellschaft für Psychiatrie und Nervenkrankheiten» auf diese diagnostische Möglichkeit hin und zeigte dabei das erste derartige Röntgenbild.

14 Der englische Physiologe Victor Alexander Haden Horsley (1857-1916) schlug als erster vor, den Hypophysentumor operativ zu entfernen.

${ }^{15}$ Fedor Krause (1856-1937).

${ }^{16}$ Hermann Schloffer (1868-1937), damaliger Leiter der chirurgischen Klinik der Universität Innsbruck. 
17 Julius Hochenegg (1859-1940) operierte am 14. Februar 1908 in Wien nach der Schlofferschen Methode (endonasal) als erster einen Fall von Akromegalie, und zwar mit ausgezeichnetem Erfolg.

${ }^{18}$ Oskar Hirsch: (geb. 1877) Über endonasale Operationsmethoden bei Hypophysis-Tumoren mit Bericht über 12 operierte Fälle (Vortrag gehalten am III. Internationalen LaryngoRhinologen-Kongress zu Berlin [30. August-2. September 1911]).

${ }^{19}$ M. Bleuler: Endokrinologische Psychiatrie, in Psychiatrie der Gegenwart, Bd I/1, 2. Aufl. Berlin-Heidelberg: Springer 1979, S.262-267.

${ }^{20}$ P. H. Althoff: Diagnostik und Therapie der Akromegalie, Sandorama 1980/III, S. 8-9.

${ }^{21}$ Henry-Alfred Henrot (1838-1919) publizierte 1877 einen Fall, den der Autor irrtümlicherweise als Myxoedem ansah.

${ }^{22}$ C. Benda: Über den normalen Bau und patholog. Veränderungen der Hypophysis cerebri, Verh. der physiol. Gesellsch. in Berlin. 1899/1900.

18. Februar 1900.

${ }^{23}$ Joh. Dom. Santorini, Observ. anatom. Venet. 1724.

\section{Literaturverzeichnis}

Abderhalden, R.: CIBA-Zeitschrift, Sondernummer: Die innere Sekretion, Basel 1951, Band 11, Nr. 124, S. 4572-4579.

Althoff, P.-H.: Diagnostik u. Therapie der Akromegalie, Sandorama 1980/III, Sandoz A.G., Basel 1980.

Baumgartner, L.: A note on the Fritzsche-Klebs discription of Akromegaly (1884). Bull. Hist. Med., 1940, 8, p. 446-460, illus. bibliog.

Bleuler, M.: Endokrinologische Psychiatrie, Stuttgart: Thieme 1954, S.119-142.

- Endokrinologische Psychiatrie, in Psychiatrie der Gegenwart, Bd. I/1, 2. Aufl., BerlinHeidelberg: Springer 1979, S. 258-342.

Cyon, E. von: Die Gefäßdrüsen als regulatorische Schutzorgane des Zentral-Nervensystems, Berlin 1910, S. 130-134.

Frankcom, G. and Musgrave, J. H.: The Irish Giant: Gerald Duckworth und Co. Ldt., The Old Piano Factory, 43 Gloucester Crescent, London N. W.1, 1976.

Fritzsche, Chr.F., und Klebs, E.: Ein Beitrag zur Pathologie des Riesenwuchses, Leipzig 1884. Fröhlich, A.: Ein Fall von Tumor der Hypophysis cerebri ohne Akromegalie, Wiener klinische Rundschau, XV.Jahrgang, Nr.47, Wien 1901. (Nach einer am 12.October 1901 in der Wanderversammlung des Vereins für Psychiatrie u. Neurologie in Wien gehaltenen Demonstration). S. 883-886.

Hirsch, O.: Über endonasale Operationsmethoden bei Hypophysis-Tumoren mit Bericht über 12 operierte Fälle. Berliner klinische Wochenschrift, 48, 1911, S. 1933-1935.

Landolt, A.M.: «Akromegalie, die häßlichste Krankheit der Welt», Neue Zürcher Zeitung Nr.234 vom 6.10.1976.

Laquer, F.: Hormone u. innere Sekretion, V: Die Hypophyse, Dresden u. Leipzig 1934, S. 206-212.

Marie, P.: Sur deux cas d'acromégalie, Revue de Médecine, 6.année, Nr.4, Paris 1886, S. 297-333. 
Minkowski, O.: Über einen Fall von Akromegalie, Berliner klinische Wochenschrift, 24, 1887, S. 371-374.

Oswald, A.: Die Erkrankung der endokrinen Drüsen. I. Die Hypophyse, Bern 1949, S.28-63. Rathke, Hch.: Über die Entstehung der Glandula pituitaria, Archiv für Anatomie u. Physiologie u. wissenschaftliche Medizin, Johannes Müller (Hrsg.), Leipzig 1838, S. $482-485$.

Schönwetter, H.P.: Zur Vorgeschichte der Endokrinologie, Inaugural-Dissertation, Juris Verlag, Zürich 1968, S.32-35.

Solheid, W.: Zur Geschichte der Hypophyse, Inaugural-Dissertation, Düsseldorf 1937, S. 40-42 u. 54-55.

Stüssi Hch.: «Die Heimsuchung des Peter Rhyner», Neujahrsbote für das Glarner Hinterland, 13. Jahrgang, 1979, S. 101-105.

Vesalius, A.: Bruxellensis, scholae, medicorum, Patauinae professoris, de Humani corporis fabrica, Libri septem, Basileae 1543, S. 654.

Zondek, B., und Aschheim, S.: Das Hormon des Hypophysenvorderlappens. Klinische Wochenschrift, 6. Jahrgang, Nr. 6, 1927, S.248-252.

- Das Hormon des Hypophysenvorderlappens (Darstellung, chemische Eigenschaften, biologische Wirkungen). Klinische Wochenschrift, 7. Jahrgang, Nr. 18, 1928, S. 831-835.

\section{Verdankung}

An dieser Stelle möchte ich Frau Prof.Dr.med.Esther Fischer-Homberger und Herrn PD Dr.phil. Heinz Balmer herzlich danken für ihre Mithilfe bei der Suche nach Literatur und biographischen Angaben. Danken möchte ich auch meiner Sekretärin, Frau E. Tinner, die mit großer Hingabe das Manuskript geschrieben hat.

\section{Summary}

Though the hypertrophy of the hypophysis has been described long ago as a pathological and anatomical symptom of acromegaly (by Fritzsche, Klebs, Pierre Marie, and others), it was Oscar Minkowski who first recognized in 1887 the etiological significance of this fact. Usually only Pierre Marie is cited in relation to acromegaly, although the first exact description of this new syndrome has been given by the first hospital physician of Glaris, Christian Friedrich Fritzsche, an by the pathologist in Zürich, Edwin Klebs.

Dr. med. Hans Peter Schönwetter

Neugasse 55

CH-9000 St. Gallen 